\title{
Prevalence of sarcopenia in patients with non alcoholic fatty liver disease
}

\begin{abstract}
Introduction: Nonalcoholic fatty liver disease (NAFLD) in the western world is associated with obesity and sarcopenia, thus, the aim of this study was to evaluate lumbar muscle mass in NAFLD patients and compare it to patients without NAFLD.
\end{abstract}

Methods: A retrospective review of computed tomography (CT) of patients with biopsy proven NAFLD from 01/2012-01/2017 was conducted. The control (C) group was comprised of patients who had undergone abdominal CT for diagnosis of renal stone. For all subjects, CT image at the third lumbar vertebrae was examined to measure area of psoas and lumbar paraspinal muscles. Data were analyzed by the use of Chi square test and Student's T-test, as appropriate.

Results: 83 patients with NAFLD and 83 control subjects were identified of whom 44 and 41 were women, respectively, were included. Average body mass index was $30.3 \pm 5.6 \mathrm{~kg} /$ $\mathrm{m}^{2}$ in NAFLD vs $30.6 \pm 6.6$ in the control group. Patients with NAFLD had lower psoas and lumbar paraspinal muscle mass than those in the control group $(\mathrm{p}<0.001)$.

Conclusion: NAFLD is associated with sarcopenia in the population of East Harlem, New York City. Further studies regarding the genetic predisposition to sarcopenia in this population is warranted.

Keywords: nonalcoholic fatty liver disease, computed tomography, body mass index, non alcoholic steatohepatitis, C-reactive protein
Volume II Issue 4 - 2020

\author{
Priyanka Yerragorla,' Pankaj Nepal,' Divya \\ Nekkalapudi,' Laura Andreias,' Shady \\ Ahmed,' Ritu Dabas, ' Ali Mousavi, ${ }^{2}$ Morcos \\ Morcos, ${ }^{2}$ Hussein Matari, ${ }^{2}$ Jennifer Harley, ${ }^{3}$ \\ Nora V Bergasa' \\ 'Department of Medicine, New York Medical College, USA \\ 2Department of Radiology, New York Medical College, USA \\ ${ }^{3}$ Department of Gastroenterology, New York Medical College, \\ USA
}

Correspondence: Nora V Bergasa, Department of Internal Medicine, Woodhull Medical and Mental Health Center, USA, Tel 212-423-677I, Email Nora.Bergasa@icloud.com

Received: July 12, 2020 | Published: August I4, 2020
Abbreviations: NAFLD, nonalcoholic fatty liver disease; CT, computed tomography; NASH, nonalcoholic steatohepatitis; BMI, body mass index

\section{Introduction}

"Sarcopenia is the age-associated loss of skeletal muscle mass and function. Sarcopenia is a complex syndrome that is associated with muscle mass loss alone or in conjunction with increased fat mass. The causes of sarcopenia are multi-factorial and can include disuse, changing endocrine function, chronic diseases, inflammation, insulin resistance, and nutritional deficiencies". ${ }^{1}$ There is emerging evidence to support an association between low muscle mass, i.e. sarcopenia, with or without fat loss, and non alcoholic fatty liver disease (NAFLD), a condition also associated with, metabolic syndrome. ${ }^{2}$ Furthermore, the presence of sarcopenia in patients with NAFLD is independently associated with advanced stages of liver disease including nonalcoholic steatohepatitis (NASH), fibrosis, and cirrhosis. ${ }^{1,3}$

The prevalence of sarcopenia in NAFLD or vice versa is unknown. Lumbar muscle mass is an indirect correlate of sarcopenia. ${ }^{5}$ Accordingly, the aim of this study was to explore the presence of sarcopenia in patients with NAFLD by the indirect measure of lumbar muscle mass.

\section{Methods}

This was a retrospective review of computed tomography (CT) of patients with biopsy proven NAFLD from 01/2012-01/2017, period chosen because of feasibility. The control group was comprised of patients who had undergone abdominal CT for the diagnosis of renal stones. Excluded from the control group were patients who had abnormal liver profile, liver disease, and any cancer. The radiologists calculated muscle mass and reviewed the CT scans of patients in the control group to exclude the radiographic suggestion of hepatic steatosis. For all subjects, CT image at third lumbar vertebrae was examined to measure area of psoas and lumbar paraspinal muscles (psoas, quadratus lumborum, and erector spinae muscles). Data were analyzed by the use of IBM SPSS Statistics 24 . Categorical variables were analyzed using Chi square test and continuous variables using Student's T-test. The study was approved by the Institutional Review Board.

\section{Results}

83 patients with NAFLD and $83 \mathrm{C}$ were identified (Table 1). The composition of the groups was similar in terms of age, gender, and body mass index (BMI); the mean age of the patients in the NAFLD group was $48 \pm 12$ years, with 39 subjects ( $46.9 \%$ ) being men versus the control group, in which the mean age of the subjects was $46 \pm 14$ years, and $42(50 \%)$ were men. The mean BMI was $30.3 \pm 5.6 \mathrm{~kg} / \mathrm{m}^{2}$ in the NAFLD group vs $30.6 \pm 6.6$ in the control. Patients with NAFLD had a lower psoas and lumbar paraspinal muscle mass than those of patients in the control group $(\mathrm{p}<0.001)$ (Table 1). CT images of a patient with NAFLD and a control subject highlighting of lumbar paraspinal muscle areas are shown in Figures 1 and 2, respectively.

\section{Discussion}

In this study, the group of patients with NAFLD had a significantly lower psoas and lumbar paraspinal muscle mass than the control subjects, suggesting an association between fatty liver and sarcopenia. NAFLD is a spectrum of histological liver findings that include hepatic steatosis, non alcoholic steatohepatitis (NASH), fibrosis, and cirrhosis. ${ }^{1,6}$ Lean NAFLD is hepatic steatosis in all its spectrum in 
individuals with normal BMI according to racial and ethnic groups; however, most patients with NAFLD have metabolic syndrome or some of its components including central obesity, type 2 diabetes, hypertension, and dyslipidemia. ${ }^{1}$

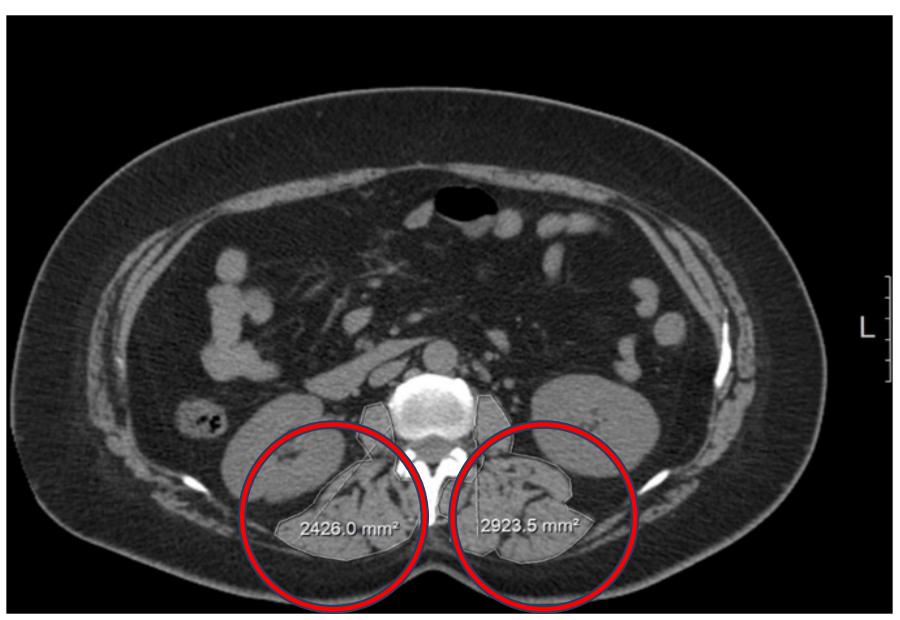

Figure I Image of a CT scan of a patient with non alcoholic fatty liver disease and sarcopenia, as suggested by decreased psoas muscle area (circle).

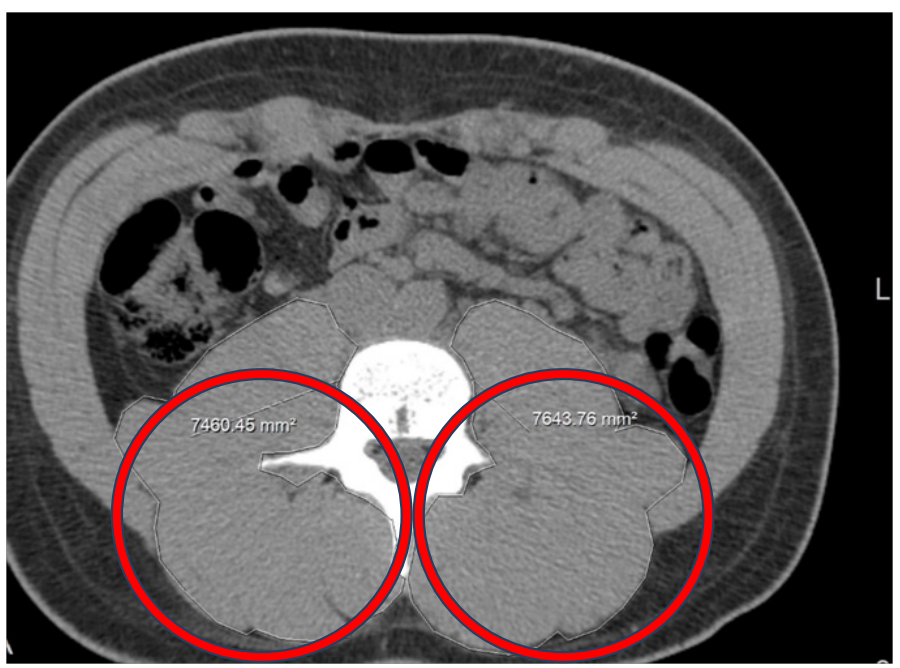

Figure 2 Image of a CT scan of a patient from the control group, i.e. without NAFLD. The circle indicates the psoas muscles.

\begin{tabular}{|c|c|c|c|}
\hline Variable & $\begin{array}{l}\text { Subjects } \\
(n=83)\end{array}$ & $\begin{array}{l}\text { Controls } \\
(n=83)\end{array}$ & P-value \\
\hline Gender (M) & $39(46.9 \%)$ & $42(50.6 \%)$ & 0.7 \\
\hline Age (yrs) & $48 \pm 12$ & $46 \pm 14$ & 0.32 \\
\hline BMI $\left(\mathrm{Kg} / \mathrm{m}^{2}\right)$ & $30.3 \pm 5.6$ & $30.6 \pm 6.6$ & $<0.001 *$ \\
\hline $\begin{array}{l}\text { R psoas muscle area } \\
\left(\mathrm{mm}^{2}\right)\end{array}$ & $616 \pm 294$ & $858 \pm 267$ & $<0.001 *$ \\
\hline $\begin{array}{l}\text { L Psoas muscle area } \\
\left(\mathrm{mm}^{2}\right)\end{array}$ & $643 \pm 299$ & $835 \pm 277$ & $<0.001 *$ \\
\hline $\begin{array}{l}\mathrm{R} \text { paraspinal muscles area } \\
\left(\mathrm{mm}^{2}\right)\end{array}$ & $3260 \pm 931$ & $4030 \pm 865$ & $<0.001 *$ \\
\hline $\begin{array}{l}\text { L paraspinal muscles area } \\
\left(\mathrm{mm}^{2}\right)\end{array}$ & $3318 \pm 925$ & $3927 \pm 820$ & $<0.00 I^{*}$ \\
\hline
\end{tabular}

*=statistically significant, Continuous variables are expressed as means \pm SD
Impaired function and failure of pancreatic beta cells is the main core problem in type 2 diabetes, however, insulin resistance by skeletal muscle is considered to be the initiating factor and is apparent years prior to the clinical manifestation of diabetes by hyperglycemia. ${ }^{8}$ Accordingly, the link between muscle, and loss of muscle mass, i.e. sarcopenia, and NAFLD may be insulin resistance meaning that sarcopenia would be a contributing and even a causative factor of hepatic steatosis. The mechanisms by which insulin resistance contributes to NAFLD is speculative and includes de novo lipogenesis by the activation of sterol regulatory element binding protein, a decrease in adiponectin, and the release of proinflammatory cytokines from adipocytes. ${ }^{9}$ We favor the hypothesis that an inflammatory milieu propitiated by insulin resistance, in part originated from sarcopenia, may be the link between the latter and NAFLD. In this context, fatty liver and sarcopenia were reported to correlate with high sensitivity C-reactive protein (hsCRP), a marker of inflammation..$^{10}$ In addition, skeletal muscle mass index had a negative relationship with triglyceride and total body fat, the increase of which can be found in an important proportion of patients with NAFLD. ${ }^{11}$ In addition, obesity, a feature of the metabolic syndrome often found in association with NAFLD is also associated with an inflammatory environment, supporting inflammation as a contributing mechanism to the pathogenesis of NAFLD. ${ }^{12}$

\section{Conclusion}

In summary, sarcopenia is associated with NAFLD, and may be an etiologic factor of this prevalent hepatopathy in the population of East Harlem, New York City, which is comprised mainly of subjects of the Hispanic Ethnicity. Further exploration, perhaps by conducting genetic studies is warranted.

\section{Acknowledgments}

None.

\section{Conflicts of interest}

Author declares that there are no conflicts of interest.

\section{Funding}

None.

\section{References}

1. Younossi ZM, Koenig AB, Abdelatif D, et al. Global epidemiology of nonalcoholic fatty liver disease-Meta-analytic assessment of prevalence, incidence, and outcomes. Hepatology. 2016;64(1):73-84.

2. Petta S, Ciminnisi S, Di Marco V, et al. Sarcopenia is associated with severe liver fibrosis in patients with non-alcoholic fatty liver disease. Aliment Pharmacol Ther. 2017;45(4):510-518.

3. Koo BK, Kim D, Joo SK, et al. Sarcopenia is an independent risk factor for non-alcoholic steatohepatitis and significant fibrosis. $J$ Hepatol. 2017;66(1):123-131.

4. Lee K, Shin Y, Huh J, et al. Recent Issues on Body Composition Imaging for Sarcopenia Evaluation. Korean J Radiol. 2019;20(2):205-217.

5. Sheka AC, Adeyi O, Thompson J, et al. Nonalcoholic Steatohepatitis: A Review. JAMA. 2020;323(12):1175-1183.

6. Younes R, Bugianesi E. NASH in Lean Individuals. Semin Liver Dis. 2019;39(1):86-95. 
7. Fielding RA, Vellas B, Evans WJ, et al. Sarcopenia: an undiagnosed condition in older adults. Current consensus definition: prevalence, etiology, and consequences. International working group on Sarcopenia. Journal of the American Medical Directors Association. 2011;12(4):249-256.

8. Warram JH, Martin BC, Krolewski AS, et al. Slow glucose removal rate and hyperinsulinemia precede the development of type II diabetes in the offspring of diabetic parents. Annals of Internal Medicine. 1990;113(12):909-915.

9. Khan RS, Bril F, Cusi K, et al. Modulation of Insulin Resistance in Nonalcoholic Fatty Liver Disease. Hepatology. 2019;70(2):711-724.
10. Moutachakkir M, Lamrani Hanchi A, Baraou A, et al. Immunoanalytical characteristics of C-reactive protein and high sensitivity C-reactive protein. Ann Biol Clin (Paris). 2017;75(2):225-229.

11. Hong HC, Hwang SY, Choi HY, et al. Relationship between sarcopenia and nonalcoholic fatty liver disease: the Korean Sarcopenic Obesity Study. Hepatology. 2014;59(5):1772-1778.

12. Bastard JP, Maachi M, Lagathu C, et al. Recent advances in the relationship between obesity, inflammation, and insulin resistance. Eur Cytokine Netw. 2006;17(1):4-12. 